\title{
SAITOH'S INEQUALITY AND OPIAL'S INEQUALITY
}

\begin{abstract}
AKIRA YAMADA
Abstract. We prove an elementary integral inequality which extends a norm inequality of Saitoh concerning absolutely continuous functions on an interval of the real axis. Our inequality immediately yields well-known Opial-type inequalities.
\end{abstract}

Mathematics subject classification (2010): 26D10.

Keywords and phrases: Reproducing kernel; Opial's inequality; geometrically convex.

\section{REFERENCES}

[1] N. Aronszajn, Theory of reproducing kernels, Trans. Amer. Math. Soc. 68 (1950), 337-404. MR 14:479c

[2] P. R. BEesack, Elementary proofs of some Opial-type integral inequalities, J. Analyse Math. 36 (1979), 1-14 (1980). MR 81g:26006

[3] P. R. BeEs ACK AND K. M. DAs, Extensions of Opial's inequality, Pacific J. Math. 26 (1968), 215232. MR 39:385

[4] J. CALVert, Some generalizations of Opial's inequality, Proc. Amer. Math. Soc. 18 (1967), 72-75. MR 34:4433

[5] E. K. Godunova And V. I. Levin, An inequality of Maroni (Russian), Mat. Zametki 2 (1967), 221-224. MR 36:333

[6] J. MatkowsKi, $L^{p}$-like paranorms, in Selected topics in functional equations and iteration theory (Graz, 1991), Grazer Math. Ber. 316 (1992), 103-138. MR 94g:39010

[7] D. S. Mitrinović, "Analytic inequalities", Die Grundlehren der mathematischen Wissenschaften, Band 165, Springer-Verlag, New York-Berlin 1970, xii+400 pp. MR 43:448

[8] Z. Opial, Sur une inégalité, Ann. Polon. Math. 8 (1960), 29-32. MR 22:3772

[9] S. S AiтoH, Theory of reproducing kernels and its applications, Pitman Research Notes in Mathematics Series, 189, Longman Scientific \& Technical, Harlow (1988), x+157 pp. MR 90f:46045

[10] S. SAITOH, Integral transforms, reproducing kernels and their applications, Pitman Research Notes in Mathematics Series, 369, Longman, Harlow (1997), xiv+280 pp. MR 98k:46041

[11] S. SAITOH, Natural norm inequalities in nonlinear transforms, in General inequalities, 7 (Oberwolfach, 1995), 39-52, Internat. Ser. Numer. Math., 123, Birkhaüser, Basel, 1997. MR 98j:46023

[12] M. Yamada And S. SAItoh, Identifications of non-linear systems, J. Comput. Math. Optim. 4 (2008), no. 1, 47-60. MR 2009b:93051 